\title{
Significance of Extra-Esophageal Symptoms in Pediatric Gastroesophageal Reflux Disease
}

\author{
Andro Košec ${ }^{10}$ Orjena Žaja ${ }^{2} \quad$ Filip Matovinović ${ }^{1}$ \\ 1 Department of Otorhinolarygology and Head and Neck Surgery, \\ University Clinical Hospital Centre Sestre Milosrdnice, Zagreb \\ University School of Medicine, Zagreb, Croatia \\ 2 Department of Pediatrics, University Clinical Hospital Centre Sestre \\ Milosrdnice, Zagreb University School of Medicine, Zagreb, Croatia \\ ${ }^{3}$ Department of Otorhinolaryngology, Mostar University Hospital, \\ Mostar, Bosnia and Herzegovina
}

\author{
Boris Jelavić ${ }^{3}$ Tomislav Baudoin ${ }^{1}$
}

Int Arch Otorhinolaryngol 2020;24(4):e472-e476.

\begin{abstract}
Address for correspondence Andro Košec, MD, PhD, FEBORL-HNS, Department of Otorhinolarygology and Head and Neck Surgery, University Clinical Hospital Centre Sestre Milosrdnice, Zagreb University School of Medicine, Vinogradska cesta 29, 10000 Zagreb, Croatia (e-mail: andro.kosec@yahoo.com).
\end{abstract}

\begin{abstract}
Keywords

- diagnostics

- obesity

- pediatric gastroesophageal reflux disease

- screening

Introduction Current practice guidelines in gastroesophageal reflux disease (GERD) often require invasive diagnostic testing.

Objective The aim of the present study was to evaluate the significance of extraesophageal symptoms and reliability of a screening risk score that is simple to use.

Methods A longitudinal retrospective single-institution cohort study. Setting: A university clinical hospital tertiary referral center. The present study enrolled pediatric patients with symptoms suggestive of GERD: epigastric pain, occasional nausea, regurgitation, tasting acid in the oral cavity, chronic cough, hoarseness of voice, frequent throat clearing. The patients underwent 24-hour esophageal pH monitoring and fiber-optic laryngoscopy. The correlations between the local findings, anamnestic and objective measurement data were analyzed.

Results The present study evaluated 89 pediatric patients. Patients with asthma presented significantly more often with adjoining gastrointestinal symptoms $(p=0.0472)$. Patients that were obese were linked to a higher rate of reports of gastrointestinal symptoms $(p=0.0495)$. After the patients had been assigned to newly developed risk groups, obesity showed to be significantly more frequent in patients placed in higher risk groups $(p<0.0001)$ for a positive GERD diagnosis.

Conclusion Patients with leading symptoms of asthma presented significantly more often with adjoining gastrointestinal symptoms. Obesity showed to be significantly more frequent in patients placed in higher risk groups for a positive GERD diagnosis.
\end{abstract}

\section{Introduction}

Pediatric gastroesophageal reflux disease (GERD) is characterized by backflow of gastric contents into the esophagus, with acid-induced and pepsin-mediated injury to the mucosa causing troublesome symptoms, affecting up to $3.3 \%$ of the pediatric population. ${ }^{1}$ The manifestations of GERD have been labeled as either esophageal or extra-esophageal syndromes (EESs).
Among the latter, atypical manifestations of GERD, including chronic cough and pediatric laryngopharyngeal reflux (PLPR) have been considered significant by general physicians, pediatricians and otorhinolaryngologists. ${ }^{2}$ In particular, there are increasing evidence linking the growing prevalence of PLPR among GERD patients. ${ }^{3}$ There is evidence that PLPR is associated with rhinosinusitis, laryngitis, pneumonia, and asthma in children, but the comorbidities are still frequently overlooked received

June 8, 2019

accepted

October 20, 2019
DOI https://doi.org/

10.1055/s-0039-3402437. ISSN $1809-9777$.
Copyright (e 2020 by Thieme Revinter

Publicações Ltda, Rio de Janeiro, Brazil
License terms

()ㅇㅇ $\Theta \circledast$ 
by otorhinolaryngologists and pediatricians. ${ }^{4}$ Children with GERD often experience extra-esophageal symptoms that can be intermittent. ${ }^{5}$ Controversies remain regarding the confirmation of the diagnosis of extra-esophageal GERD manifestations in general. At present, insufficient data are available on the assessment of GERD symptoms and on the characteristics of the symptom complex in these patients. In October 2009, the North American Society for Pediatric Gastroenterology, Hepatology, and Nutrition (NASPGHAN) and the European Society for Pediatric Gastroenterology, Hepatology, and Nutrition (ESPGHAN) published new clinical practice guidelines for the diagnosis and management of reflux in the pediatric population, updating and unifying their previous guidelines as a means of improving uniformity of practice and quality of patient care. One of the most frequent deviations from the NASPGHAN/ESPGHAN recommendations concerned the use of diagnostic modalities. Previously published studies show symptom description to be unreliable and nonspecific. ${ }^{6}$ Current practice guidelines in GERD often require invasive diagnostic testing. The aim of the present study was to evaluate the prevalence of extra-esophageal symptoms and the reliability of a novel screening score that is simple to use.

\section{Methods}

The present study was conducted as a retrospective longitudinal cohort study. It encompassed pediatric patients who presented with extra-esophageal GERD symptoms (epigastric pain, nausea, regurgitation, acid in the oral cavity, chronic cough, dysphonia, frequent throat clearing) to the pediatric gastroenterologist first, who were then evaluated by an otorhinolaryngologist. The 24-hour dual-probe monitoring procedure was performed in all pediatric patients with extra-esophageal symptoms clearly present in their patient history as part of a department diagnostic protocol. Initially, data from 121 pediatric patients who underwent 24 -hour esophageal $\mathrm{pH}$ monitoring and fiber optic laryngoscopy were evaluated. Out of these, 32 patients were excluded from the study due to incomplete treatment data and informed consent forms. The correlations between local findings and anamnestic data and objective data obtained by 24-hour dual-probe monitoring were analyzed. The present study was conducted from January 1st, 2007, to December 31st, 2012, in a tertiary pediatric and otorhinolaryngology referral center.

The present study and its protocol were designed and conducted adhering to the 1983 Helsinki Declaration, and informed consent was obtained from all of the patients' parents and legal guardians. After fasting, 24-hour double-probe $\mathrm{pH}$ monitoring was performed with the use of a nasal catheter and $\mathrm{pH}$ recorder(Flexisoft III, Oakfield Instruments Ltd, Witney, UK). ${ }^{7}$ All of the patients were on their usual diet during monitoring. A positive diagnosis of GERD was observed if there was a $\mathrm{pH}$ value $<4$ for more than $5 \%$ of the total recording time. Three or more episodes of $\mathrm{pH}<4$ in the proximal probe and distal probe simultaneously, or $1 \%$ of the total recording time showing $\mathrm{pH}<4$ in the proximal probe were accepted as PLPR. The measures calculated included reflux, Boix-Ochoa and DeMeester-Johnson indexes, and number of acid reflux events lasting $>5$ minutes. The measurements were performed by the same pediatric gastroenterologist. The otorhinolaryngologic examination was performed by the same otorhinolaryngologist to reduce interobserver variability, through fiber-optic laryngoscopy (4-mm flexible optic fiber, Karl Storz, Tuttlingen, Germany) that assessed the upper airway from the nasal vestibule to the infraglottic area. The patients' parents gave written informed consent for the diagnostic testing.

Obesity was factored in as an independent predictive factor if the patient's body mass index (BMI) was at least two standard deviations (SDs) above the World Health Organization (WHO) growth reference median. The diagnosis of asthma was established through spirometry examinations (reduced forced expiratory flow higher than $25-75 \%$ of the Forced Vital Capacity [FVC]).

The statistical analysis was performed using the MedCalc software ( MedCalc Software, Ostend, Belgium), version 11.2.1. The data for the cohort were expressed as ratios due to the fact that it was composed of less than 100 patients $(n=89)$. The associations between variables were assessed using the Fisher exact test, Mann-Whitney test and Kruskal-Wallis test for nonparametric paired samples. All of the tests of statistical significance were performed using a two-sided 5\% type-I error rate.

\section{Results}

The present study included 89 pediatric patients with a median age of 12 . There were 56 girls and 33 boys. Out of these patients, 52 had positive diagnostic criteria for GERD, and 50 of these 52 had positive criteria for PLPR. When gastrointestinal comorbidity was analyzed, 15/89 complained of epigastric pain, 14/89 had occasional nausea, 10/89 experienced regurgitation, and 3/89 complained of tasting acid in the oral cavity.

Data showed that the moderate and severe forms of GERD were most prevalent, and that boys tended to have GERD significantly more often than girls (Mann-Whitney U test, $p<0.0001$ ), and scored significantly higher on the $\mathrm{pH}$ monitoring tests (Mann-Whitney U test, $p<0.0001$ ). Patients with leading symptoms of asthma and asthma + cough were statistically significantly correlated with higher GERD grades (Kruskal-Wallis test, $p=0.0493$ ). They also presented significantly more often with adjoining gastrointestinal symptoms in our cohort (Kruskal-Wallis test, $p=0.0472$ ). Patients that had obesity as a comorbidity factor were linked to a significantly higher rate of reports of gastrointestinal symptoms (Mann-Whitney U test, $p=0.0495$ ).

A risk score encompassing clinical findings, gastrointestinal symptoms, comorbidities and obesity was then developed. After the patients had been assigned to the newly-developed risk groups, a significant correlation with a positive GERD diagnosis was found among the groups (Kruskal-Wallis test, $p=0.0262$ ) (-Table 1, - Fig. 1). When adding obesity to the scoring table as an independent factor, an even higher rate of corresponding risk distribution among the risk groups and GERD severity categories was evident (Kruskal-Wallis test, $p=0.0109$ ) ( - Fig. 2). Obesity alone showed to be significantly more frequent in patients placed in higher risk categories 
Table 1 Gastroesophageal reflux disease (GERD) diagnostic probability scoring tool

\begin{tabular}{|l|l|l|}
\hline $\begin{array}{l}\text { Upper respiratory } \\
\text { symptoms } \\
\text { and local findings } \\
\text { (oropharyngoscopy } \\
\text { and } \\
\text { nasal fiber optic } \\
\text { laryngoscopy) }\end{array}$ & $\begin{array}{l}\text { Chronic cough } \\
\text { Chronic throat clearing } \\
\text { Dysphonia } \\
\text { Halitosis } \\
\text { Oropharyngeal redness } \\
\text { and granulation } \\
\text { Posterior laryngitis } \\
\text { Vocal nodules }\end{array}$ & $\begin{array}{l}\text { If present } \\
+1\end{array}$ \\
\hline $\begin{array}{l}\text { Gastrointestinal } \\
\text { symptoms }\end{array}$ & $\begin{array}{l}\text { Nausea } \\
\text { Gastric or epigastric pain } \\
\text { Vomiting } \\
\text { Malnourishment }\end{array}$ & $\begin{array}{l}\text { If present } \\
+1\end{array}$ \\
\hline Comorbidity & $\begin{array}{l}\text { Asthma } \\
\text { Recurrent laryngitis }\end{array}$ & $\begin{array}{l}\text { If present } \\
+1\end{array}$ \\
\hline Obesity & $\begin{array}{l}\text { Body-mass index } \\
\text { >2 standard } \\
\text { deviations above the } \\
\text { World Health Organization } \\
\text { growth reference median }\end{array}$ & $\begin{array}{l}\text { If present } \\
+1\end{array}$ \\
\hline Score & $\begin{array}{l}\text { Low GERD risk: 0/1 points } \\
\text { Moderate GERD risk: } \\
2 \text { points } \\
\text { High GERD risk: } 3-4 \text { points }\end{array}$ & \begin{tabular}{l} 
Total \\
\hline
\end{tabular} \\
\hline
\end{tabular}

(Kruskal-Wallis test, $p<0.0001$ ) ( - Fig. 3). Finally, when analyzing the patients with gastrointestinal symptoms, it became apparent that they were more frequently allotted to higher risk groups (Kruskal-Wallis test, $p<0.0001$ ) ( Fig. 4).

\section{Discussion}

Although a clinical entity that has been increasingly recognized in recent years, pediatric GERD and its associated symptom complex continues to pose more questions than answers. It is often connected with failure to thrive, chronic cough, dysphonia and laryngeal spasm. ${ }^{8}$ The extra-esophageal symptom complex is often diagnosed by noting atypical laryngoscopic findings: erythema, edema, ventricular obliteration, postcricoid hyperplasia and pseudosulcus. However,

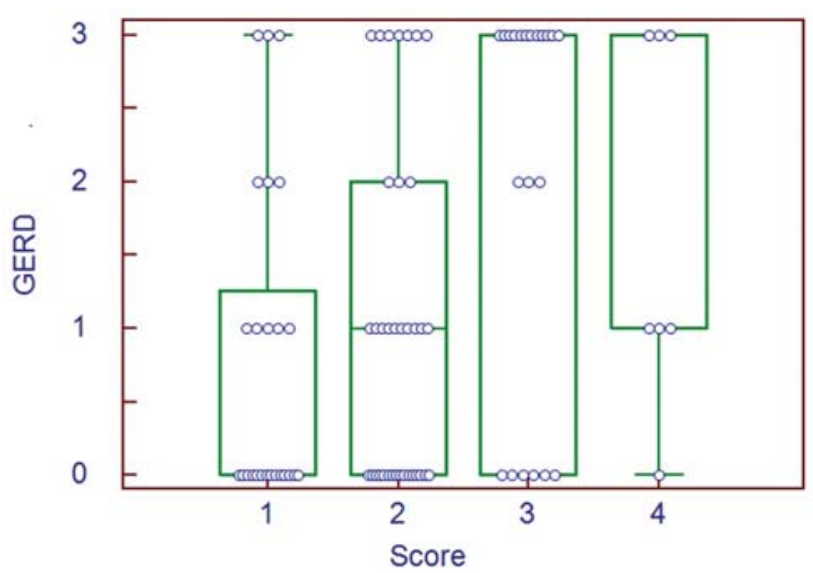

Fig. 1 Correlation between rising gastroesophageal reflux disease (GERD) grades and the newly proposed screening score (KruskalWallis test; $p=0.0109 ; n=89$ ).

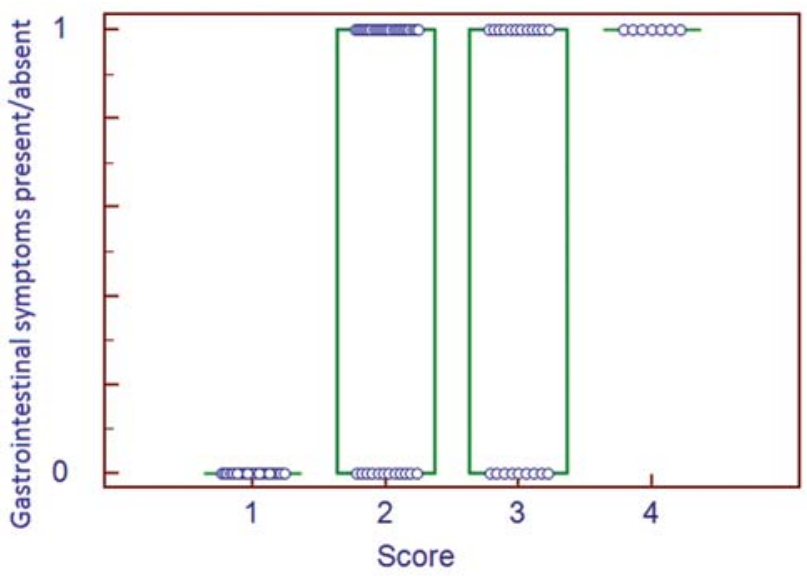

Fig. 2 Correlation between positive gastrointestinal symptoms and rising risk scores in the newly proposed screening score (KruskalWallis test; $p<0.0001 ; n=89$ ).

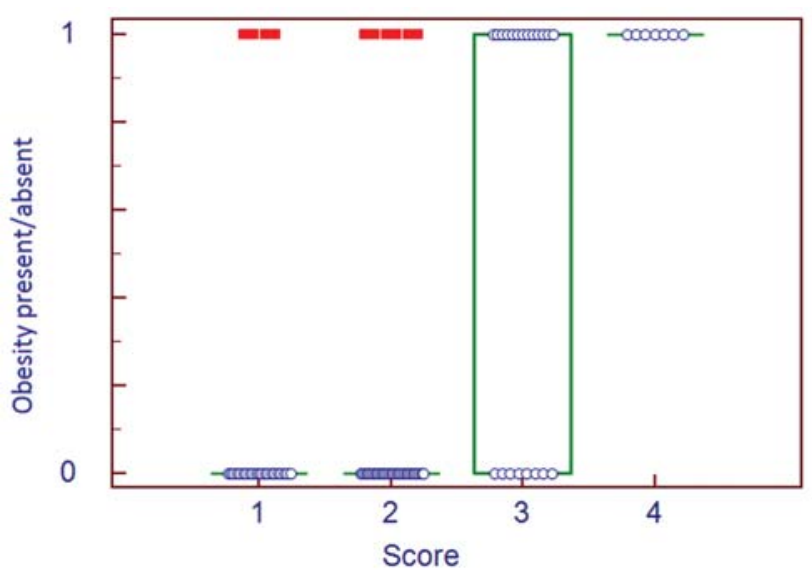

Fig. 3 Correlation between obesity as an independent risk factor and rising risk scores in the newly proposed screening score (KruskalWallis test; $p<0.0001 ; n=89$ ).

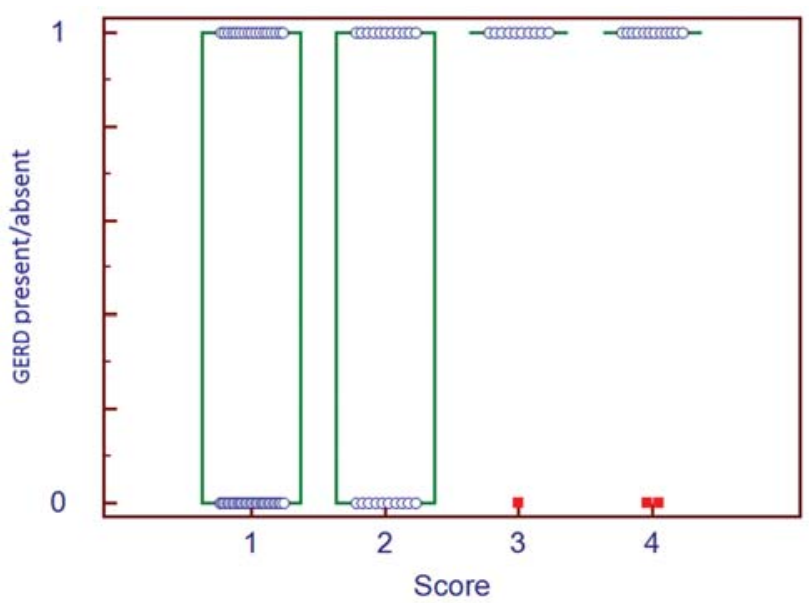

Fig. 4 Correlation between GERD diagnosis through 24-hour $\mathrm{pH}$ monitoring and probability scoring using the newly proposed screening score (Kruskal-Wallis test; $p=0.0017 ; n=89$ ). 
these findings are also common in healthy volunteers, and this largely limits their diagnostic value. ${ }^{9}$ Gastroesophageal reflux disease is considered an important factor in perpetuating laryngeal inflammation. ${ }^{10}$ Few studies shed light on the characteristics of reflux episodes and adjoining gastrointestinal symptoms in those patients. Numerous controversies encompass the clinical manifestations, diagnostics, interpretation of findings and treatment. ${ }^{11,12}$ This is even more of a challenge when considering that the most frequent deviation from the NASPGHAN/ESPGHAN recommendations are diagnostic modalities for GERD. According to a recent study, ${ }^{13}$ the vast majority of pediatricians do not perform specific testing and diagnose solely based on patient history-taking, even in young children and infants. A study ${ }^{14}$ surveyed North American pediatric health care providers in 2007 and found that $31 \%$ of them diagnosed GERD without performing specific testing, irrespective of age. A study ${ }^{15}$ performed in 2012 found a similar attitude among $59 \%$ of the surveyed French pediatricians. Even when extra-esophageal manifestations have been suspected based on 24-hour dual-probe esophageal $\mathrm{pH}$ monitoring results, it may still be inaccurate, since its sensitivity is low and the incidence of false-negative results is as high as $25 \%$ to $50 \%{ }^{16-18}$ The limitations of diagnosing GERD solely based on patient symptoms, without the 24-hour esophageal pH testing, remain a major hindrance in achieving a timely diagnosis and subsequent symptom control. ${ }^{19}$ In addition, many GERD patients present with atypical symptoms. ${ }^{20}$ Most frequently, respiratory symptoms are present but difficult to describe objectively. A variety of otorhinolaryngologic symptoms may be identified, with a suggested correlation with asthma. ${ }^{21}$ Our study diagnosed 50 out of 52 GERD patients (total cohort: $n=89$ ) with PLPR through 24-hour esophageal probe $\mathrm{pH}$ monitoring. Esophageal probe $\mathrm{pH}$ monitoring was performed in all of the patients because they were referred to our institution by pediatric gastroenterologists who had previously suspected a possible GERD/PLPR diagnosis. In 42 out of 89 patients, the following was noted: epigastric pain (15/89), nausea (14/89), regurgitation (10/89), and acid aftertaste in the oral cavity (3/89) (-Fig. 1). Patients with asthma and chronic cough (48/89) showed a noted propensity for higher GERD levels. Out of 50 patients with a positive GERD diagnosis, 25 had reported gastrointestinal symptoms. Boys tended to have more advanced GERD levels, according to 24-hour esophageal $\mathrm{pH}$ monitoring results, but accounted for only $1 / 3$ of the patients. All of the patients had respiratory symptoms at the initial evaluation, with the most frequent being an injected and granulated oropharynx accompanied by posterior laryngitis (54/89). To date, very few studies have correlated the clinical presentation in PLPR and GERD and the diagnostic accuracy by objective testing. Most published studies favor fiber-optic laryngoscopy as a method of upper-airway clinical assessment, but few attempts have been made to create a structured risk score using available respiratory and gastrointestinal findings, especially in the pediatric population..$^{10,11,17,18}$ The results of our study, as well as those of previously published studies, show that the diagnosis confirmation rate can be improved without subjecting the patient to unnecessary testing. ${ }^{22}$
With the help of data gathered in our study, patient symptoms and factors that have been correlated to extra-esophageal symptoms in our patient cohort were used to develop a risk score to reduce unnecessary testing and help administer proper and cost-effective treatment ${ }^{23}$ (-Table $\mathbf{1}$ ). When allocating the patients into risk groups, four major criteria were used; respiratory symptoms and findings (otorhinolaryngologist evaluation); gastrointestinal symptoms (gastroenterologist evaluation); respiratory comorbidity (asthma or recurrent laryngitis); and presence of obesity. The otorhinolaryngologist noted the presence of chronic cough, throat clearing, dysphonia, halitosis, oropharyngeal redness and granulation, posterior laryngitis or vocal nodules. The gastroenterologist noted the presence of nausea, gastric or epigastric pain, vomiting or malnourishment. Respiratory comorbidity was also noted by either examiner, mainly asthma or recurrent laryngitis. Obesity was factored in if the patient's BMI exceeded 2 SDs above the WHO growth reference median. ${ }^{24}$ Every group (gastrointestinal symptoms, respiratory symptoms, comorbidity and obesity) scored one point or zero points, and depending on the cumulative score (0-4), the diagnosis of GERD was considered ( $\boldsymbol{- T a b l e} \mathbf{1})$. If at least one positive symptom from the symptom group was present, a point was added. In the end, the patients were divided into 3 risk groups: patients that had a high probability score for GERD (3-4 points); patients that had a moderate probability score for GERD ( 2 points); and patients that had a low probability score for GERD ( $0-1$ point). The risk score showed that in our appropriate patient groups, 25 patients had a low risk for GERD, 36 had a moderate risk, and 28 had a high risk for GERD. The patient groups showed significant correlation with a positive GERD diagnosis, with obesity as an independent factor that improved scoring reliability (Kruskal-Wallis test, $p=0.0109$ ) (-Figs. 1, 2, 3). A strong correlation is shown with GERD grading as well, which confirms that the scoring system functions well for GERD risk stratification (Kruskal-Wallis test, $p=0.0020$ ). (-Fig. 2) A one-sheet score based on additive categories encompassing all of the benchmarks of the initial patient evaluation ensures a helpful assessment of the GERD risk, and could prove useful in the everyday clinical setting. The management of GERD in children includes lifestyle changes, pharmacologic therapy, and surgery. Among the pharmacologic agents, proton pump inhibitors (PPIs) are confirmed superior to histamine-2 receptor antagonists (H2RAs) to heal erosive esophagitis and relieve symptoms; however, the dramatic increase in the patterns of prescription of PPIs during the past several years has raised concerns related to their appropriate use and associated cost. ${ }^{25}$ Proton pump inhibitors were administered to all of the patients diagnosed with moderate and severe GERD by 24 -hour doubleprobe $\mathrm{pH}$ monitoring in our study, with favorable follow-up results. The treatment seems to demonstrate symptom reduction as well as improvements in the concomitant diseases, such as PLPR.

\section{Conclusions}

Extra-esophageal symptoms should always be considered when dealing with GERD as a chronic disease with a variety 
of presentations. The present study investigated the relationships between clinical symptoms and objective findings, and it stress the need for a comprehensive appraisal of both gastrointestinal and respiratory symptoms in the everyday clinical practice.

\section{Conflict of Interests}

The authors have no conflict of interests to declare.

\section{References}

1 Sherman PM, Hassall E, Fagundes-Neto U, et al. A global, evidencebased consensus on the definition of gastroesophageal reflux disease in the pediatric population. Am J Gastroenterol 2009;104 (05):1278-1295, quiz 1296

2 Richter JE. Extraesophageal presentations of gastroesophageal reflux disease: an overview. Am J Gastroenterol 2000;95(8, Suppl)S1-S3

3 Leone CA, Mosca F. Multicentric study: statistical correlation between clinical data and instrumental findings in laryngopharyngeal reflux: proposal for a new ENT classification of reflux. Acta Otorhinolaryngol Ital 2006;26(05):264-270

4 Zalzal GH, Tran LP. Pediatric gastroesophageal reflux and laryngopharyngeal reflux. Otolaryngol Clin North Am 2000;33(01): 151-161

5 Karkos PD, Leong SC, Apostolidou MT, Apostolidis T. Laryngeal manifestations and pediatric laryngopharyngeal reflux. Am J Otolaryngol 2006;27(03):200-203

6 Vandenplas Y, Rudolph CD, Di Lorenzo C, et al; North American Society for Pediatric Gastroenterology Hepatology and Nutrition; European Society for Pediatric Gastroenterology Hepatology and Nutrition. Pediatric gastroesophageal reflux clinical practice guidelines: joint recommendations of the North American Society for Pediatric Gastroenterology, Hepatology, and Nutrition (NASPGHAN) and the European Society for Pediatric Gastroenterology, Hepatology, and Nutrition (ESPGHAN). J Pediatr Gastroenterol Nutr 2009;49(04):498-547

7 Sarani B, Gleiber M, Evans SRT. Esophageal pH monitoring, indications, and methods. J Clin Gastroenterol 2002;34(03):200-206

8 Venkatesan NN, Pine HS, Underbrink M. Laryngopharyngeal reflux disease in children. Pediatr Clin North Am 2013;60(04):865-878

9 Vaezi MF. Laryngitis and gastroesophageal reflux disease: increasing prevalence or poor diagnostic tests? Am J Gastroenterol 2004;99 (05):786-788

10 Koufman JA, Aviv JE, Casiano RR, Shaw GY. Laryngopharyngeal reflux: position statement of the committee on speech, voice, and swallowing disorders of the American Academy of Otolaryngology-Head and Neck Surgery. Otolaryngol Head Neck Surg 2002;127 (01):32-35

11 Milstein CF, Charbel S, Hicks DM, Abelson TI, Richter JE, Vaezi MF. Prevalence of laryngeal irritation signs associated with reflux in asymptomatic volunteers: impact of endoscopic technique (rigid vs. flexible laryngoscope). Laryngoscope 2005;115(12):2256-2261

12 Bredenoord AJ, Weusten BL, Smout AJ. Symptom association analysis in ambulatory gastro-oesophageal reflux monitoring. Gut 2005;54(12):1810-1817

13 Quitadamo P, Papadopoulou A, Wenzl T, et al. European pediatricians' approach to children with GER symptoms: survey of the implementation of 2009 NASPGHAN-ESPGHAN guidelines. J Pediatr Gastroenterol Nutr 2014;58(04):505-509

14 Diaz DM, Winter HS, Colletti RB, et al; NASPGHAN/CDHNF Scientific Advisory Board. Knowledge, attitudes and practice styles of North American pediatricians regarding gastroesophageal reflux disease. J Pediatr Gastroenterol Nutr 2007;45(01):56-64

15 Martigne L, Delaage PH, Thomas-Delecourt F, Bonnelye G, Barthélémy P, Gottrand F. Prevalence and management of gastroesophageal reflux disease in children and adolescents: a nationwide crosssectional observational study. Eur J Pediatr 2012;171(12):1767-1773

16 Praud JP. Upper airway reflexes in response to gastric reflux. Paediatr Respir Rev 2010;11(04):208-212

17 Lundell LR, Dent J, Bennett JR, et al. Endoscopic assessment of oesophagitis: clinical and functional correlates and further validation of the Los Angeles classification. Gut 1999;45(02):172-180

18 Vavricka SR, Storck CA, Wildi SM, et al. Limited diagnostic value of laryngopharyngeal lesions in patients with gastroesophageal reflux during routine upper gastrointestinal endoscopy. Am J Gastroenterol 2007;102(04):716-722

19 Palmer R, Anon JB, Gallagher P. Pediatric cough: what the otolaryngologist needs to know. Curr Opin Otolaryngol Head Neck Surg 2011;19(03):204-209

20 Winter HS, Illueca M, Henderson C, Vaezi M. Review of the persistence of gastroesophageal reflux disease in children, adolescents and adults: does gastroesophageal reflux disease in adults sometimes begin in childhood? Scand J Gastroenterol 2011;46(10):1157-1168

21 Stavroulaki P. Diagnostic and management problems of laryngopharyngeal reflux disease in children. Int J Pediatr Otorhinolaryngol 2006;70(04):579-590

22 May JG, Shah P, Lemonnier L, Bhatti G, Koscica J, Coticchia JM. Systematic review of endoscopic airway findings in children with gastroesophageal reflux disease. Ann Otol Rhinol Laryngol 2011; 120(02):116-122

23 Baudoin T, Kosec A, Cor IS, Zaja O. Clinical features and diagnostic reliability in paediatric laryngopharyngeal reflux. Int J Pediatr Otorhinolaryngol 2014;78(07):1101-1106

24 Koebnick C, Getahun D, Smith N, Porter AH, Der-Sarkissian JK, Jacobsen SJ. Extreme childhood obesity is associated with increased risk for gastroesophageal reflux disease in a large population-based study. Int J Pediatr Obes 2011;6(2-2):e257-e263

25 Karkos PD, Wilson JA. Empiric treatment of laryngopharyngeal reflux with proton pump inhibitors: a systematic review. Laryngoscope 2006;116(01):144-148 